\title{
Clinical Trial Highlights - an update on previously reviewed trials
}

\author{
Kevin McFarthing PhD ${ }^{a s}$, Neha Prakash $M B B S^{b}$ and Tanya Simuni $M D^{c}$ \\ aParkinson's research advocate, Oxford, UK. \\ 'Parkinson's Disease and Movement Disorders Center, University of Connecticut School of Medicine, \\ Farmington, CT, USA \\ 'Parkinson's Disease and Movement Disorders Center, Northwestern University Feinberg School of Medicine, \\ Chicago, IL, USA \\ § To whom correspondence should be addressed at kevin.mcfarthing@gmail.com
}

\section{BACKGROUND}

The Journal of Parkinson's Disease started the Clinical Trial Highlights (CTH) section in January 2019. The objective was "to raise awareness of the clinical trial landscape in Parkinson's disease (PD), promoting discussion and progress in the conduct and outcome of studies." In addition, it was intended to be "a resource centre for academics, medics, industry and PwP, particularly those wanting to participate in clinical trials." After three years, it is useful to step back and review the progress of the trials we featured. Since the start, we have reviewed studies in seven categories. CTH has hosted two reviews of the clinical trials pipeline. It also provides a resource list covering various aspects of information on, and participating in, clinical trials in PD. The home page for CTH is at https://www.journalofparkinsonsdisease.com/clinical-trial-highlights .

\section{OVERVIEW}

Some of the trials we reviewed had already been completed at the time of our publications, but the projects were still active and were included in the review in order to give a full overview of the drug development in each area. When we exclude these previously completed studies from this current update, we have reviewed 58 studies of which 13 have been completed and six of these studies have also published results on www.clinicaltrials.gov .

Since our previously published review of each category, new trials have started and the reviewed trials have progressed. This brief update gives the current status of trials in the respective categories.

\section{CATEGORY UPDATE}

\section{Targeting $\alpha$-synuclein}

The formation of oligomers and fibrils of $\alpha$-synuclein is strongly associated with the pathology of PD. There are potential therapies under development that target the spread of multimeric forms of a $\alpha$-synuclein from cell to cell, using synthetic antibodies that recognise specific epitopes on aggregated $\alpha$-synuclein. Other small molecule-based therapies aim to prevent mis-folding or the formation of multimers. A summary of the status of therapies targeting $\alpha$-synuclein is shown in Table 1 .

\section{Antibody-based therapies}

Roche's prasinezumab, a synthetic monoclonal antibody, failed to meet its primary outcomes in the phase 2 PASADENA study (NCT03100149), a statistically significant reduction in parts I, II and III of the MDSUPDRS. However, it did show signals of efficacy on selected prespecified secondary and exploratory clinical 
endpoints and was generally well-tolerated [1]. The PASADENA study has entered a year-long extension and a new phase 2 study, entitled PADOVA (NCT04777331), that is enrolling early but treated PD participants, is now under way.

Biogen's antibody program, BIIB054 (cinpanemab), failed to meet its primary and secondary outcomes in the phase 2 SPARK study (NCT03318523). The program has been terminated. AstraZeneca's MEDI1341 has completed a single ascending dose phase 1 study (NCT03272165), and is now in a phase 1 multiple ascending dose study (NCT04449484).

Lundbeck's antibody-based LuAF82422 has completed phase 1 for PD, with results yet to be announced (NCT03611569). The company recently announced their intention to evaluate the compound in a phase 2 study for multiple system atrophy (MSA) [2].

New programs have entered the clinical stage of development. A phase 1 trial was registered for ABBV0805, a synthetic antibody against aggregated $\alpha$ - synuclein. It started in March 2020 but was withdrawn in July 2020 for "strategic considerations" (NCT04127695).

Affiris' vaccine against $\alpha$-synuclein, PD01, has been renamed ACI-7104 following the acquisition of Affiris by AC Immune, and a phase 2 study is being planned but as yet does not appear to have been registered. AC Immune have also completed a study to optimise morphomer-based $\alpha$-synuclein PET tracers (NCT05067192).

Vaxxinity's (formerly named United Neurosciences) program for UB-312 is now in phase 1 (NCT04075318).

UCB's anti- $\alpha$-synuclein antibody, UCB7853 is in early phase 1 with testing in healthy male volunteers (NCT04651153).

\section{Small molecule approaches}

The two studies on nilotinib, a c-Abl inhibitor that increases $\alpha$-synuclein clearance, at Georgetown University (NCT02954978) and Northwestern University/ MJFF (NCT03205488) both demonstrated acceptable tolerability and safety but failed to show symptomatic benefit in motor or cognitive domains. It appears that low brain penetrance was the major factor in the disappointing results, so the c-Abl kinase remains a strong target for future therapies.

Phenylbutyrate up-regulates DJ-1 and has been shown to protect dopaminergic neurons in vitro [3]. The Colorado University phase 1 study on glycerol phenylbutyrate is planned to complete in October 2021.

Squalamine inhibits the formation of aggregated $\alpha$-synuclein in pre-clinical models, and restores the function of the enteric nervous system [4]. Following a successful phase 2a trial in PD-associated constipation (RASMET; NCT03047629), Enterin has started two further phase 2 trials for ENT-01, a synthetic version of squalamine. The first is a phase $2 \mathrm{~b}$ in constipation (KARMET; NCT03781791); the other is evaluating the potential for the use of ENT-01 in PD dementia (NCT03938922).

Mannitol has been shown to inhibit $\alpha$-synuclein aggregation in pre-clinical animal models [5]. It is now in a phase 2 study (NCT03823638) although the entry on clinicaltrials.gov is of "Unknown" status.

Modag's Anle138b also modulates $\alpha$-synuclein oligomer formation and protects against dopaminergic neuronal loss in preclinical models [6]. It has completed a phase 1 dose-ranging study (NCT04208152), and is now in a phase 1 trial to assess pharmacokinetics and pharmacodynamics in PD patients (NCT04685265).

UCB and their partner Neuropore have successfully completed a phase 1 with UCB0599, an inhibitor of $\alpha$-synuclein misfolding (NCT04875962). The molecule is now in phase 2 (NCT04658186). 
Annovis Bio (formerly QR Pharma) has initiated a phase 2 trial (NCT04524351) with ANVS401 (posiphen). Pre-clinical work showed that the molecule targets neurotoxic proteins ( $\alpha$-synuclein, tau and $\beta$-amyloid) to enhance axonal transport [7].

A summary of clinical trials targeting $\alpha$-synuclein is shown in Table 1 .

Table 1 - summary of clinical trials in PD targeting $\alpha$-synuclein.

\begin{tabular}{|c|c|c|c|c|c|}
\hline SPONSOR & AGENT & $\begin{array}{l}\text { REGISTRY } \\
\text { NUMBER }\end{array}$ & PHASE & $\begin{array}{l}\text { REGISTRY } \\
\text { STATUS }\end{array}$ & COMPLETION DATE \\
\hline Biogen & BIIB054 & NCT03318523 & 2 & Terminated & 29 April 2021 \\
\hline Lundbeck & Lu AF82422 & NCT03611569 & 1 & Recruiting & 31 August 2021 (estimated) \\
\hline Roche & Prasinezumab & NCT03100149 & 2 & Active, not recruiting & 23 April 2026 (estimated) \\
\hline AstraZeneca & MEDI1341 & NCT03272165 & 1 & Completed & 31 March 2021 \\
\hline UCB & UCB7853 & NCT04651153 & 1 & Recruiting & June 2023 (estimated) \\
\hline Colorado University & $\begin{array}{l}\text { Glycerol phenyl } \\
\text { butyrate }\end{array}$ & NCT02046434 & 1 & Active, not recruiting & October 2021 \\
\hline Georgetown University & Nilotinib & NCT02954978 & 2 & Unknown & July 2020 (estimated) \\
\hline $\begin{array}{l}\text { Northwestern University/ } \\
\text { MJFF }\end{array}$ & Nilotinib & NCT03205488. & 2 & Completed & 28 September 2019 \\
\hline Abbvie & ABBV0805 & NCT04127695 & 1 & Withdrawn & July 2022 \\
\hline Vaxinnity & UB312 & NCT04075318 & 1 & Recruiting & 30 December 2022 \\
\hline Enterin & ENT-01 & NCT03781791 & 2 & Recruiting & September 2021 (estimated) \\
\hline Enterin & ENT-01 & NCT03938922 & 2 & Active, not recruiting & 25 May 2021 (estimated) \\
\hline $\begin{array}{l}\text { Hadassah Medical } \\
\text { Organisation }\end{array}$ & Mannitol & NCT03823638 & 2 & Unknown & $\begin{array}{l}31 \text { December } 2020 \\
\text { (estimated) }\end{array}$ \\
\hline Modag & Anle138b & NCT04208152 & 1 & Completed & 4 August 2020 \\
\hline Modag & Anle138b & NCT04685265 & 1 & Recruiting & June 20222 (estimated) \\
\hline UCB & UCB0599 & NCT04875962 & 1 & Completed & 19 February 2020 \\
\hline UCB & UCB0599 & NCT04658186 & 2 & Recruiting & July 2024 (estimated) \\
\hline Annovis Bio & $\begin{array}{l}\text { ANVS401 } \\
\text { (posiphen) }\end{array}$ & NCT04524351 & 2 & Active, not recruiting & $\begin{array}{l}1 \text { December } 2021 \\
\text { (estimated) }\end{array}$ \\
\hline
\end{tabular}

\section{References}

[1] https://www.michaeljfox.org/news/news-context-pasadena-study

[2] https://news.cision.com/h--lundbeck-a-s/r/lundbeck-launches-a-phase-ii-study-for-potential-newtreatment-of-multiple-system-atrophy,c3442560

[3] Zhou W, Bercury K, Cummiskey J, Luong N, Lebin J, Freed CR. Phenylbutyrate up-regulates the DJ-1 protein and protects neurons in cell culture and in animal models of Parkinson disease. J Biol Chem. 2011 Apr 29;286(17):14941-51.

[4] West CL, Mao YK, Delungahawatta T, Amin JY, Farhin S, McQuade RM, Diwakarla S, Pustovit R, Stanisz AM, Bienenstock J, Barbut D, Zasloff M, Furness JB, Kunze WA. Squalamine Restores the Function of the Enteric Nervous System in Mouse Models of Parkinson's Disease. J Parkinsons Dis. 2020;10(4):1477-1491. doi: 10.3233/JPD-202076.

[5] Shaltiel-Karyo R, Frenkel-Pinter M, Rockenstein E, Patrick C, Levy-Sakin M, Schiller A, Egoz-Matia N, Masliah E, Segal D, Gazit E. A blood-brain barrier (BBB) disrupter is also a potent $\alpha$-synuclein ( $\alpha$-syn) aggregation inhibitor: a novel dual mechanism of mannitol for the treatment of Parkinson disease (PD). J Biol Chem. 2013 Jun 14;288(24):17579-88. doi: 10.1074/jbc.M112.434787. 
[6] Levin J, Schmidt F, Boehm C, Prix C, Bötzel K, Ryazanov S, Leonov A, Griesinger C, Giese A. The oligomer modulator anle $138 \mathrm{~b}$ inhibits disease progression in a Parkinson mouse model even with treatment started after disease onset. Acta Neuropathol. 2014 May;127(5):779-80. doi: 10.1007/s00401-014-1265-3.

[7] https://www.annovisbio.com/technologies

\section{Gene therapy}

Our original review had two programs in phase 2 and two in phase 1. Of these, one phase 1 has been officially terminated (Jichi Medical University; NCT02418598), while the phase 2 for VY-AADC (Amino Acid Decarboxylase) from Voyager Therapeutics has been placed on clinical hold by the FDA, and Voyager's partner, Neurocrine, has terminated the agreement between the companies [1].

The Axo-Lenti-PD program at Axovant continues (NCT03720418), with target completion due in 2022, although the final trial end date is in 2031. The NINDS study for AAV-GDNF (Glial cell-Derived Neurotrophic Factor; NCT16211851) remains active but not recruiting, as it approaches the projected study end date of February 2022.

Since our review, two further studies have been registered. Ask Bio (formerly Brain Neurotherapy Bio) is also pursuing GDNF delivered by an AAV2 vector in phase 1 (NCT04167540). Prevail Therapeutics has PR001 in a phase $1 / 2$ trial in patients with at least one mutation in the GBA1 gene (NCT04127578). MeiraGTx appears to be still preparing for a phase 2 study with AAV-GAD (Glutamic Acid Decarboxylase), but as yet there are no registry entries for this therapy. A summary of trials of gene therapy in PD is shown in Table 2.

Table 2 - summary of clinical trials in PD using gene therapy.

\begin{tabular}{llllll}
\hline SPONSOR & AGENT & $\begin{array}{l}\text { REGISTRY } \\
\text { NUMBER }\end{array}$ & PHASE & REGISTRY STATUS & COMPLETION DATE \\
\hline $\begin{array}{l}\text { Jichi Medical } \\
\text { University }\end{array}$ & AAV-hAADC & NCT02418598 & 1 & Terminated & 31 March 2018 \\
$\begin{array}{l}\text { Voyager } \\
\text { Therapeutics }\end{array}$ & VY-AADC02 & NCT03562494 & 2 & Active, not recruiting & December 2022 (estimated) \\
$\begin{array}{l}\text { Axovant } \\
\text { NINDS }\end{array}$ & AXO-Lenti-PD & NCT03720418 & 2 & Active, not recruiting & June 2022/December 2031 (estimated) \\
$\begin{array}{l}\text { Ask Bio } \\
\text { Arevail } \\
\text { Therapeutics }\end{array}$ & AAV2-GDNF & NCT01621581 & 1 & Active, not recruiting & 1 February 2022 (estimated) \\
\hline
\end{tabular}

\section{References}

[1] https://ir.voyagertherapeutics.com/news-releases/news-release-details/voyager-therapeutics-providesupdate-nbib-1817-vy-aadc-gene-0

\section{Dyskinesia}

In the July 2019 edition, we reviewed 7 trials in detail focusing on symptomatic management of dyskinesia [1]. Three trials are ongoing, two have been discontinued, and two are complete. Since our initial review, three more trials for symptomatic treatment for dyskinesia have been initiated.

Addex therapeutics have been developing dipraglurant, an allosteric modulator of the metabotropic glutamate 5 receptor (mGluR5), targeting dyskinesia. Since their completion of a phase 2a trial with results published in 
2016, the company has initiated two clinical trials, NCT04857359 and NCT05116813 [2-4]. NCT04857359 is a Phase $2 \mathrm{~b} / 3$, double blind, randomized, placebo-controlled study aiming to evaluate the efficacy of dipraglurant for dyskinesia in PwP receiving levodopa. The trial is of 12 weeks' duration measuring changes to UDysRS. After 12 weeks, participants will have the option to join NCT05116813, an open label extension study that will evaluate long term safety and tolerability of the drug over 12 months by monitoring the incidence of adverse events. Both studies have started recruitment and are estimating completion in December, 2022 and August, 2023 for the open label extension and placebo-controlled studies respectively.

No updates are available regarding the eltoprazine trials. The Hôpitaux de Paris trial evaluating buspirone for dyskinesia continues to recruit and is expected to complete by end of 2021 or beginning of 2022. The trial with JM-010, a combination therapy of buspirone and zolmitriptan, continues to recruit participants and has extended the completion date to March 2022 from the original date of June 2021. NCT02589340, studying the effect of buspirone in combination with amantadine on dyskinesia was terminated in March 2021 due to low enrolment ( $n=6$, estimated enrolment was for 15 participants) [5]. Having enrolled 23 patients, the phase 2 study investigating pridopidine was terminated in November 2020 citing COVID-19 as the reason [6]. The molecule continues to be explored for Huntington's disease and amyotrophic lateral sclerosis.

IRL790, a novel dopamine receptor D3 antagonist, completed the phase 2 study in June 2019, as anticipated, enrolling a total of 75 participants. Formal results have not been published yet. Based on the company website and clinicaltrial.gov updates, the study achieved a clinically meaningful effect on dyskinesia as measured by Hauser diaries and UPDRS scale, which were the secondary outcomes for the study. The updates have not mentioned the effect on the primary outcome measure of UDysRS [7, 8]. The molecule has since been advanced to a longer (3 months) and larger (140 participants) phase $2 \mathrm{~b} / 3$ study evaluating its effect on dyskinesia (NCT04435431). The trial has been recruiting since October 2020 and is estimated to complete by January 2022 [9]. The primary outcome will measure change in the ON-time without troublesome dyskinesia based on $24 \mathrm{hr}$ Hauser diary entries instead of UDysRS, likely due to not meeting the primary end point in the Phase 2a study [7-9]. Since the last review, IRL790 has been granted the International Non-proprietary Name (INN), mesdopetam [8].

Two of the new trials are exploring molecules affecting NMDA receptors. NCT04912115, sponsored by PharmaTher Inc. is a multi-center, phase 2, randomized, double-blind, prospective trial evaluating the effects of intravenous infusion of ketamine on dyskinesia in participants with PD over a period of 8 weeks. The study includes an active control arm with intravenous midazolam infusion. It is estimated to complete by December 2021 [10]. NCT04147949, sponsored by VistaGen Therapeutics, Inc., is a randomized, double-blind, placebocontrolled, crossover, proof of concept, phase 2 study exploring the efficacy and safety of AV-101 (L-4chlorokynurenine) in PwP with dyskinesia, over a period of 2 weeks. AV-101 is a prodrug for 7-chlorokynurenic acid, which is a potent and specific NMDA receptor glycine site antagonist with poor CSF penetrance [11].

A summary of clinical trials for dyskinesia is shown in Table 3.

Table 3 - summary of clinical trials in PD for dyskinesia.

\begin{tabular}{llllll}
\hline SPONSOR & AGENT & $\begin{array}{l}\text { REGISTRY } \\
\text { NUMBER }\end{array}$ & PHASE & $\begin{array}{l}\text { REGISTRY } \\
\text { STATUS }\end{array}$ & COMPLETION DATE \\
\hline $\begin{array}{lllll}\text { Coeptis/Elto Pharma } \\
\text { Hôpitaux de Paris }\end{array}$ & Eltoprazine & NCT02439125 & 2 & Unknown & December 2017 \\
Contera/ Bukwang & $\begin{array}{l}\text { JM-010 (buspirone and } \\
\text { zolmitriptan) }\end{array}$ & NCT02617017 & 3 & Unknown & June 2018 \\
Oregon University & $\begin{array}{l}\text { Buspirone and } \\
\text { amantadine }\end{array}$ & NCT02589340 & 1 & Terminated & 23 February 2021 \\
$\begin{array}{l}\text { Integrative Research } \\
\text { Laboratories }\end{array}$ & IRL-790 & NCT03368170 & 2 & Completed & 12 June 2019
\end{tabular}


Table 3 - (Continue)

\begin{tabular}{llllll}
\hline SPONSOR & AGENT & $\begin{array}{l}\text { REGISTRY } \\
\text { NUMBER }\end{array}$ & PHASE & $\begin{array}{l}\text { REGISTRY } \\
\text { STATUS }\end{array}$ & COMPLETION DATE \\
\hline Prilenia & Pridopidine & NCT03922711 & 2 & Terminated & 22 July 2020 \\
Pharmather & Ketamine & NCT04912115 & 2 & $\begin{array}{l}\text { Enrolling by } \\
\text { invitation }\end{array}$ & 31 December 2021 (estimated) \\
VistaGen Theapeutics & AV-101 & NCT04147949 & 2 & Not yet recruiting & April 2024 (estimated) \\
Addex Pharma & $\begin{array}{l}\text { ADX48621 } \\
\text { (dipraglurant) }\end{array}$ & NCT04857359 & $2 / 3$ & Recruiting & August 2023 (estimated) \\
Addex Pharma & $\begin{array}{l}\text { ADX48621 } \\
\text { (dipraglurant) }\end{array}$ & NCT05116813 & $2 / 3$ & Recruiting & December 2022 (estimated) \\
\hline
\end{tabular}

\section{References}

[1] McFarthing K, Prakash N, Simuni T. CLINICAL TRIAL HIGHLIGHTS - DYSKINESIA. Journal of Parkinson's Disease. 2019;9(3):449-465. doi:10.3233/JPD-199002

[2] Tison F, Keywood C, Wakefield M, et al. A Phase 2A Trial of the Novel mGluR5-Negative Allosteric Modulator Dipraglurant for Levodopa-Induced Dyskinesia in Parkinson's Disease. Movement disorders : official journal of the Movement Disorder Society. 2016;31(9):1373-1380. doi:10.1002/MDS.26659

[3] Dipraglurant (ADX48621) for the Treatment of Patients With Parkinson's Disease Receiving Levodopabased Therapy - Full Text View - ClinicalTrials.gov. Accessed November 21, 2021. https://www. clinicaltrials.gov/ct2/show/NCT04857359?term=Dipraglurant\&draw=2\&rank=3

[4] Open-label Safety Study of Dipraglurant (ADX48621) in Patients With Parkinson's Disease Receiving Levodopa-based Therapy - Full Text View - ClinicalTrials.gov. Accessed November 21, 2021. https:// www.clinicaltrials.gov/ct2/show/NCT05116813?term=Dipraglurant\&draw=2\&rank=1

[5] History of Changes for Study: NCT02589340. Accessed November 21, 2021. https://www.clinicaltrials. gov/ct2/history/NCT02589340?A=1\&B=6\&C=Side-by-Side\#StudyPageTop

[6] History of Changes for Study: NCT03922711. Accessed November 21, 2021. https://clinicaltrials.gov/ct2/ history/NCT03922711?B=6\&A=7\&C=Side-by-Side\#StudyPageTop

[7] Efficacy and Tolerability of IRL790 in Parkinson's Disease Dyskinesia - Study Results - ClinicalTrials. gov. Accessed November 21, 2021. https://clinicaltrials.gov/ct2/show/results/NCT03368170?term=NCT $03368170 \&$ draw $=2 \&$ rank $=1$

[8] Mesdopetam (IRL790) - IRLAB. Accessed November 21, 2021. https://www.irlab.se/project-portfolio/ irl-790/

[9] A Clinical Study of Mesdopetam in Patients With Parkinson's Disease Experiencing Levodopa Induced Dyskinesia - Full Text View - ClinicalTrials.gov. Accessed November 21, 2021. https://clinicaltrials.gov/ ct2/show/NCT04435431?term=mesdopetam \&draw $=2 \&$ rank $=1$

[10] Randomized, Double-Blind, Active Placebo-Controlled Study of Ketamine to Treat Levodopa-Induced Dyskinesia - Full Text View - ClinicalTrials.gov. Accessed November 21, 2021. https://clinicaltrials. gov/ct2/show/NCT04912115?term=NCT04912115\&draw=2\&rank=1

[11] Levodopa-Induced Dyskinesia (LID) :: VistaGen Therapeutics, Inc. (VTGN). Accessed November 21, 2021. https://www.vistagen.com/pipeline/av-101/levodopa-induced-dyskinesia 


\section{Levodopa and dopamine agonist infusion therapies}

Despite the two currently available infusion therapies, there is scope for the development of more sophisticated and user-friendly alternatives for drug delivery. In our previous issue on infusion therapies, we had reviewed 6 trials [1]. Five trials are ongoing and one has been completed. In the interim, two new trials have been initiated and one of them has already been concluded.

Abbvie has been working on subcutaneous (sc) infusion of ABBV-951, now known as foslevodopa/foscarbidopa. ABBV-951 is a solution of these prodrugs for levodopa/carbidopa (LD/CD) which has been proven to have a stable LD exposure via the sc route [2]. NCT03781167 is the phase 3 efficacy trial of ABBV-951 in PwP. The recruitment status of the trial has been updated to active, not recruiting. Results and further information are not yet available.

Since the last review, Abbvie also initiated and recently concluded a 12-week, phase 3, randomized, doubleblind, double-dummy, active-controlled study of continuous sc infusion of ABBV-951 in patients with advanced Parkinson's disease. The study was first posted on clinicaltrials.gov in May 2020 (NCT04380142). In October 2021, Abbvie announced statistically significant positive results from the study [3]. At week 12, continuous 24 $\mathrm{hr} /$ day sc infusion of ABBV-951 improved ON time by $2.72 \mathrm{hr}$ as compared to oral LD/CD (0.92hr). Similarly, the OFF time reduced by $2.75 \mathrm{hr}$ with ABBV-951 and by $0.96 \mathrm{hr}$ among those on oral LD/CD. The benefits were noted as early as 1 week after starting infusion. While most of the adverse events (AE) are reported to be mild to moderate, $21.6 \%$ discontinued treatment with ABBV-951 as compared to $1.5 \%$ in the active control arm. Common AE included infusion site AE, dyskinesia, motor fluctuation, hallucination, balance disorder, falls, constipation, and peripheral swelling. The results are expected to be published soon.

Neuroderm's ND0612 sc infusion (NCT04006210) continues to recruit and has extended its study completion date to October 2023. It has also increased anticipated enrolment to 380 from 300. Rennes University's EARLY-PUMP study continues to recruit and has extended its study completion date to June 2025. US WorldMed's INFUS-ON trial of continuous apomorphine infusion pump continues to recruit patients. Supernus Pharmaceuticals, Inc has acquired US WorldMed's CNS portfolio including the apomorphine infusion pump now labelled as SPN-830. Supernus filed an NDA for SPN-830 based on previous trials but is working on pump optimisation.

IPO-001, a phase 1 pharmacokinetic study of Infudopa SubC ${ }^{\mathrm{TM}}$ and Infudopa IntraV ${ }^{\mathrm{TM}}$ compared to LD/ CD intestinal gel, completed in April 2020. According to Dizlin Pharmaceutical's press release the study demonstrated similar plasma levodopa levels with Infudopa SubC ${ }^{\mathrm{TM}}$ compared to LCIG and non-inferiority with respect to plasma level fluctuations. Both formulations are reported to provide similar motor control compared to LCIG. Mild to moderate infusion site reactions were noted with the sc route which was expected [4]. Results and publication are awaited. Dizlin intends to file for marketing authorization of Infudopa IntraV ${ }^{\mathrm{TM}}$ in Europe and USA in 2023 [5]. The company has declared a future clinical tolerability study but no further details are available.

Lillie University Hospital in France is conducting a proof-of-concept clinical phase 1/2b study (NCT04332276) which is currently recruiting participants. The study will evaluate the feasibility, safety first, and then the effectiveness of continuous dopaminergic stimulation by cerebroventricular administration of A-dopamine for advanced PD. This comes after a successful pre-clinical study where intracerebroventricular administration of the drug demonstrated improved motor symptoms without tachyphylaxis and dyskinesia with high dose [6]. A-dopamine, which is dopamine prepared in anaerobic conditions to avoid oxidation, will be stored in an intraabdominal pump expected to be refilled once in 2 weeks. The study is estimated to complete by March 2023.

A summary of clinical trials for infusion therapies is shown in Table 4. 
Table 4 - summary of clinical trials in PD for infusion therapies.

\begin{tabular}{|c|c|c|c|c|c|}
\hline SPONSOR & AGENT & $\begin{array}{l}\text { REGISTRY } \\
\text { NUMBER }\end{array}$ & PHASE & REGISTRY STATUS & COMPLETION DATE \\
\hline Abbvie & ABBV-951 & NCT3781167 & 3 & Active, not recruiting & 27 September 2022 (estimated) \\
\hline $\begin{array}{l}\text { Mitsubishi Tanabe/ } \\
\text { Neuroderm }\end{array}$ & ND0612 & NCT04006210 & 3 & Recruiting & 30 October 2023 (estimated) \\
\hline Rennes University & EARLY PUMP & NCT02864004 & 3 & Recruiting & 3 September 2024 (estimated) \\
\hline US World Meds & INFUS-ON & NCT02339064 & 3 & Active, not recruiting & September 2021 \\
\hline Dizlin & Infudopa & NCT03419806 & 1 & Completed & 20 April 2020 \\
\hline Lille University Hospital & DIVE & NCT04332276 & $1 / 2$ & Recruiting & March 2023 (estimated) \\
\hline
\end{tabular}

\section{References}

[1] Prakash N, McFarthing K, Simuni T. Clinical Trial Highlights - Infusion Therapies. Journal of Parkinson's Disease. 2020;10(1):5-17. doi:10.3233/JPD-199005

[2] Rosebraugh M, Liu W, Neenan M, Facheris MF. Foslevodopa/Foscarbidopa Is Well Tolerated and Maintains Stable Levodopa and Carbidopa Exposure Following Subcutaneous Infusion. Journal of Parkinson's disease. 2021;11(4):1695-1702. doi:10.3233/JPD-212813

[3] AbbVie Announces ABBV-951 (Foslevodopa/Foscarbidopa) Showed Improvement in Controlling Motor Fluctuations Compared to Oral Levodopa/Carbidopa Medication in Pivotal Phase 3 Trial in Patients with Advanced Parkinson's Disease | AbbVie News Center. Accessed November 23, 2021. https://news.abbvie. $\mathrm{com} /$ news/press-releases/abbvie-announces-abbv-951-foslevodopafoscarbidopa-showed-improvementin-controlling-motor-fluctuations-compared-to-oral-levodopacarbidopa-medication-in-pivotal-phase-3trial-in-patients-with-advanced-parkinsons-disease.htm

[4] Positive final results from pharmacokinetic study (PK) with Infudopa SubC ${ }^{\mathrm{TM}}$ and Infudopa IntraV ${ }^{\mathrm{TM}}$ (IPO-001) - Dizlin. Accessed November 24, 2021. https://www.dizlin.se/positive-final-results-frompharmacokinetic-study-pk-with-infudopa-subc-and-infudopa-intrav-ipo-001/

[5] Products in development - Dizlin. Accessed November 24, 2021. https://www.dizlin.se/products-indevelopment/

[6] Moreau C, Rolland AS, Pioli E, et al. Intraventricular dopamine infusion alleviates motor symptoms in a primate model of Parkinson's disease. Neurobiology of disease. 2020;139. doi:10.1016/J. NBD.2020.104846

\section{GLP-1 agonists}

The repurposing of GLP-1 agonists from diabetes to PD, which we reviewed in April 2020, has a rich pipeline of trials, with one each in phase 1 and phase 3 , and six in phase 2 . Three of the phase 2 trials are due to complete at the end of 2021, so we expect results to be announced in the first half of 2022. No further trials for GLP-1 agonists have been added to the registries. A summary of clinical trials for GLP-1 agonists in PD is shown in Table 5.

Table 5 - summary of clinical trials in PD targeting for GLP-1 agonists. 


\begin{tabular}{llllll}
\hline SPONSOR & AGENT & $\begin{array}{l}\text { REGISTRY } \\
\text { NUMBER }\end{array}$ & PHASE & $\begin{array}{l}\text { REGISTRY } \\
\text { STATUS }\end{array}$ & COMPLETION DATE \\
\hline University College, London & Exenatide & NCT04232969 & 3 & Recruiting & 30 April 2024 (estimated) \\
$\begin{array}{l}\text { Stockholm Healthcare } \\
\text { Services/Karolinska Institute } \\
\text { Peptron }\end{array}$ & Exenatide & $\begin{array}{l}\text { EUDRA CT 2019- } \\
000732-26\end{array}$ & Not stated & Not stated \\
PT320 & NCT04269642 & 2 & Active, not recruiting & $\begin{array}{l}31 \text { December 2021 } \\
\text { (estimated) }\end{array}$ \\
Neuraly & Exenatide & NCT03456687 & 1 & Active, not recruiting & May 2022 (estimated) \\
Cedars Sinai & NLY-01 & NCT04154072 & 2 & Recruiting & December 2022 (estimated) \\
Oslo University & Liraglutide & NCT02953665 & 2 & Active, not recruiting & December 2021 (estimated) \\
& Semaglutide & NCT03659682 & 2 & Not yet recruiting & $\begin{array}{l}31 \text { December 2024 } \\
\text { (estimated) }\end{array}$ \\
\hline Toulouse University Hospital & Lixisenatide & NCT03439943 & 2 & Active, not recruiting & December 2021 (estimated) \\
\hline
\end{tabular}

\section{PD Cognition}

There is a significant unmet need for developing therapies for symptomatic and disease modification for PD cognitive impairment (CI) and PD dementia (PDD). We have reviewed 8 clinical studies in different stages and targets aimed to improve cognition in PwP with CI [1]. Three studies have been completed while the remaining five are ongoing. There are four new studies under this category since our last review.

Anavex ${ }^{\circledR 2-73 ~(b l a r c a m e s i n e) ~ i s ~ a n ~ o r a l l y-a v a i l a b l e ~ s i g m a-1 ~ r e c e p t o r ~(S 1 R) ~ a c t i v a t o r ~ w h i c h ~ i s ~ b e i n g ~ e v a l u a t e d ~}$ for PD dementia in a phase 2, multi-center, double-blind, randomized, placebo-controlled trial. The study is now complete and enrolled 132 participants in a 1:1:1 ratio into either treatment arm of 30mg, 50mg ANAVEX ${ }^{\circledR} 2-73$, or placebo. The results have not been published but according to the Anavex life sciences press release, the study showed positive results in both cognitive and motor domains. It met its primary cognitive endpoints, which were CDR system Continuity of Attention (CoA) $(p=0.029)$ and CDR system Power of Attention (PoA) $(p=0.015)$. The study also met its secondary motor endpoints which included MDS-UPDRS Part III $(p=0.024)$ and MDS-UPDRS total $(p=0.038)$. At the end of 14 weeks, the adjusted mean difference in MDS-UPDRS Total score was $-14.51(p=0.034)$ with the high dose treatment arm compared to placebo [2]. The data will be submitted to FDA to seek regulatory guidance.

NMDA modulation has been the target of several medications for CI. NYX-458 by Aptinyx, and DAAOI-P by China Medical University are two drugs modulating the NMDA system and continue to recruit. NCT02914366 evaluating ambroxol for PDD continues with recruitment as well. It was estimated to complete by December 2021 but no further updates are available.

The study completion date for NYX-458 has been extended to December 2022. The trial has made a few changes since our last update. The inclusion criteria have been extended to include dementia with Lewy bodies (DLB). The study initially intended to have three experimental arms evaluating $10 \mathrm{mg}, 30 \mathrm{mg}$, or $100 \mathrm{mg}$ daily dose of NYX-458. The modifications indicate that it will only be comparing $30 \mathrm{mg}$ of the drug against placebo. The inclusion criteria have been updated to include PD with CI or DLB, both with MoCA between 15-25.

LY3154207 (Mevidalen) is a novel human D1R subtype-selective positive allosteric modulator that has been developed by Eli Lilly and Company. PRESENCE (NCT03305809) was a phase 2, placebo-controlled, study evaluating the effectiveness of different doses of mevidalen over a period of 12 weeks. The study has completed and results were anticipated at the American Academy of Neurology 2021 annual meeting [3]. The data have been released to the clinicaltrials.gov registry and are yet to be peer-reviewed. The study failed to achieve its 
primary outcome, which was Continuity of Attention (CoA) Composite Score of the Cognitive Drug Research Computerized Cognition Battery (CDR-CCB). Among the long list of secondary outcomes evaluating the cognitive and psychiatric domains, statistically significant mild improvement was noted with high dose (75mg) in the Alzheimer's Disease Cooperative Study-Clinician Global Impression of Change (ADCS-CGIC) Score, Epworth sleepiness scale, and MDS-UPDRS (I-III) mostly. AE were more in the higher dose arm. Falls were the most common AE followed by fatigue, dizziness, and nausea [4].

University of Michigan's proof of concept trial (NCT04497168) exploring if citalopram therapy could delay visuospatial cognitive decline in PD continues with recruitment. No updates have been posted for EnterinENT-01 which is still active, not recruiting (NCT03938922).

While studied extensively for PDD, there is limited evidence of the efficacy of donepezil in PD-MCI. Yonsei University explored this in their two-arm, open-label, non-randomized trial investigating the effect of donepezil on PD-MCI over 48 weeks. Results were published in February 2021 and failed to reach the primary outcome [5]. Of the 80 enrolled participants, the study result included 21 from the treatment arm and 29 as controls. There was no statistically significant change to any of the cognitive scales utilized after 48 weeks of treatment with donepezil. Short evaluation duration, high dropout rates and non-randomized design could be a few of the reasons that contributed to negative results. The conversion rate from PD-MCI to PDD was notably lower at $4 \%$, though this seems to be largely influenced by follow up duration and small sample size. Even though no clinical change was noted, the correlation with EEG demonstrated that donepezil may modulate and enhance cholinergic function in PD-MCI, mainly localized to the medial temporal region. Perhaps, further studies of longer duration and larger dataset may help understand if modulating the cholinergic system has any meaningful clinical benefit for PD-MCI.

Four new studies targeting PD cognition have been posted since our last review. CuraSen Therapeutics, Inc. is targeting the adrenergic system for PD cognition. It is evaluating the cognitive benefits and pharmacodynamic effects of co-administration of CST-103/CST-107 in a phase 2, randomized, placebo-controlled, double-blind, crossover trial (NCT04739423). The study will include approximately 40 participants with PD-MCI with REMsleep behavior disorder, MCI, and DLB or PD-dementia. The study is currently recruiting and anticipated to finish by 2022. CST-103 is a beta-2 adrenoceptor agonist, which was shown to increase cerebral blood flow in PD-MCI in a recent phase $1 \mathrm{~b}$ trial [7]. CST-107 is a beta blocker with minimal brain penetration and is being co-administered with CST-103 to counter the peripheral AE of increased heart rate, tremor, and palpitations. In the phase $1 \mathrm{~b}$ trial of CST-103, co-administration with CST-107 did not have any effect on the CST-103 induced changes to cerebral blood flow.

Eisai Inc. has been developing E2027, a selective phosphodiesterase (PDE) 9 inhibitor. It is currently in a phase 2, open-label study (NCT04764669) to evaluate the pharmacodynamic effects, efficacy, safety, and tolerability of E2027 in participants with DLB or PD Dementia, with or without amyloid co-pathology. The study anticipates enrolling 32 participants and is estimated to complete in January 2022.

PD-MIND (NCT04810104) is an international, multi-center, randomized, double-blind, placebo-controlled, parallel-group, phase 2a study of AZD0328, a selective $\alpha 7$ nicotinic receptor agonist, in PD-MCI. The study will enrol 160 participants with PD-MCI assigned to either placebo or active treatment for 12 weeks. It is anticipated to complete in June 2022. NCT04643327 is a proof of concept double-blind, randomised-controlled within-subject crossover trial evaluating the efficacy of levetiracetam for PD-MCI. The study is estimated to complete by December 2023.

Athira Pharma's ATH-1017 is based on Hepatocyte Growth Factor (HGF) and will be administered subcutaneously in a phase 2 study (NCT04831821). The study is planning to enrol 75 participants with PDD or DLB and is double blind, randomized and placebo-controlled. The treatment duration is six months. 
The Central South University in China is assessing the potential for sulphoraphane, extracted from broccoli sprouts, in a phase 2 study with two primary outcome measures, the MATRICS Cognitive Consensus Battery and MDS-UPDRS (NCT05084365). Treatment time is 6 months and the design is randomized, double-blind and placebo-controlled, with enrolment of 100 patients.

A summary of clinical trials in PD cognition is shown in Table 6.

Table 6 - summary of clinical trials for PD cognition.

\begin{tabular}{llllll}
\hline SPONSOR & AGENT & $\begin{array}{l}\text { REGISTRY } \\
\text { NUMBER }\end{array}$ & PHASE & $\begin{array}{l}\text { REGISTRY } \\
\text { STATUS }\end{array}$ & COMPLETION DATE \\
\hline Anavex & Anavex 2-73 & NCT03774459 & 2 & Completed & 30 September 2020 \\
Aptinyx & NYX-458 & NCT04148391 & 2 & Recruiting & 30 December 2022 (estimated) \\
$\begin{array}{l}\text { China Medical } \\
\text { University Hospital }\end{array}$ & DAAOI-P & NCT04470037 & 2 & Recruiting & July 2022 (estimated) \\
$\begin{array}{l}\text { Lawson Health Research } \\
\text { Institute }\end{array}$ & Ambroxol & NCT02914366 & 2 & Recruiting & December 2021 (estimated) \\
Lilly & LR3154207 & NCT03305809 & 2 & Completed & 10 July 2020 \\
University of Michigan & Citalopram & NCT04497168 & 2 & Recruiting & September 2025 (estimated) \\
Yonsei University & Donepezil & NCT02450786 & 2 & Completed & August 2019 \\
Enterin & ENT-01 & NCT03938922 & 1 & Active, not recruiting & 25 May 2021 (estimated) \\
Curasen & CST-103/CST-107 & NCT04739423 & 2 & Recruiting & March 2022 (estimated) \\
Eisai & E2027 & NCT04764669 & 2 & Active, not recruiting & 22 January 2022 \\
King's College London & AZD0328 & NCT04810104 & 2 & Not yet recruiting & June 2022 (estimated) \\
Queensland University & Levetiracetam & NCT04643327 & 2 & Recruiting & December 2023 (estimated) \\
Athira Pharma & ATH-1017 & NCT04831281 & 2 & Not yet recruiting & March 2023 (estimated) \\
Central South University & Sulphoraphane & NCT05084365 & 2 & Not yet recruiting & 31 December 2022 (estimated) \\
\hline & & & &
\end{tabular}

\section{References}

[1] Prakash N, McFarthing K, Simuni T. Clinical Trial Highlights - Parkinson's Disease Cognition. Journal of Parkinson's Disease. 2021;11(1):9-30. doi:10.3233/JPD-209006

[2] Anavex Life Sciences Announces ANAVEX®2-73 Improved both Primary Cognitive and Second MDS-UPDRS. Accessed November 25, 2021. https://www.anavex.com/post/anavex-life-sciencesannounces-anavex-2-73-improved-both-primary-cognitive-and-second-mds-updrs

[3] Biglan K, Munsie L, Svensson K, et al. Results of the Presence Study Evaluating Mevidalen for the Treatment of Lewy Body Dementia (LBD) (2028). Neurology. 2021;96(15 Supplement).

[4] A Study of LY3154207 in Participants With Dementia Due to Lewy Body Dementia (LBD) Associated With Idiopathic Parkinson's Disease (PD) or Dementia With Lewy Bodies (DLB) - Study Results ClinicalTrials.gov. Accessed November 24, 2021. https://www.clinicaltrials.gov/ct2/show/results/NC T03305809? term $=$ NCT03305809\&draw $=2 \&$ rank $=1$

[5] Baik K, Kim SM, Jung JH, et al. Donepezil for mild cognitive impairment in Parkinson's disease. Scientific Reports. 2021;11(1):4734. doi:10.1038/S41598-021-84243-4

[6] CuraSen Therapeutics Presents Phase 1b Clinical Data with CST-103 Demonstrating Significant Increases in Cerebral Blood Flow in Patients with Mild Cognitive Impairment or Parkinson's Disease | CuraSen. Accessed November 24, 2021. https://www.curasen.com/curasen-therapeutics-presents-phase-1bclinical-data-with-cst-103-demonstrating-significant-increases-in-cerebral-blood-flow-in-patientswith-mild-cognitive-impairment-or-parkinsons-disease/ 


\section{Kinase inhibitors}

Our review of kinase inhibitors was published in April 2021 so we would not expect to see major change in the composition of the trials targeting protein kinases. A summary of clinical trials for kinase inhibitors in PD is shown in Table 7.

Table 7 - summary of clinical trials in PD for kinase inhibitors.

\begin{tabular}{llllll}
\hline SPONSOR & AGENT & $\begin{array}{l}\text { REGISTRY } \\
\text { NUMBER }\end{array}$ & PHASE & REGISTRY STATUS & COMPLETION DATE \\
\hline Biogen & BIIB094 & NCT03976349 & 1 & Recruiting & 29 September 2023 (estimated) \\
Denali & DNL151 & NCT04557800 & 1 & Completed & 19 February 2021 \\
Il Yang Pharm & Radotinib & NCT04691661 & 2 & Not yet recruiting & 13 April 2022 (estimated) \\
$\begin{array}{l}\text { Sun Pharma Advanced } \\
\begin{array}{l}\text { Research Company Limited } \\
\text { 1st Biotherapeutics }\end{array}\end{array}$ & K0706 & NCT03655236 & 2 & Recruiting & March 2023 (estimated) \\
Inibikase Therapeutics & FB-101 & NCT04165837 & 1 & Recruiting & 30 June 2020 (estimated) \\
\hline
\end{tabular}

\section{Phase 3 in focus}

Since the start of the CTH section, we have covered twelve phase 3 studies in focus, ten symptomatic and two potentially disease modifying therapies. Six of the covered studies have been completed, and six continue with recruitment.

STEADY-PD III was a Phase 3, parallel group, placebo-controlled 36 months study evaluating the efficacy of isradipine $10 \mathrm{mg}$ daily as a disease modifying agent in early PD. The primary outcome looked at change in ON state UPDRS I-III from baseline to 36 months. The study enrolled 336 participants with $95 \%$ completion rate but failed to demonstrate slowing of disease progression [1].

Intec Pharma's gastric retentive Accordion Pill ${ }^{\mathrm{TM}}$ Carbidopa/Levodopa (AP-CD/LD) was being evaluated in a phase 3 study for its safety and efficacy in advanced PD. Though the results have not been published, a news press release in 2019 announced that the study did not demonstrate superiority over $\mathrm{C} / \mathrm{L}$ in reducing motor fluctuations in advanced PD patients [2]. Results of Gocovri EASE LID and EASE LID 3 trials have already been covered in our previous CTH issue [3]. The results were statistically significant and showed a clinically significant improvement in $\mathrm{ON}$ time without troublesome dyskinesia and a concomitant reduction in time with troublesome dyskinesia.

Amneal has been developing another long-acting LD/CD, IPX203 for advanced PD to reduce motor fluctuations. The results from two initial phase 2 studies of IPX203 were promising. The Phase 3, multi-center, randomized, double-blind, double-dummy, active-controlled, parallel-group study (RISE-PD) has completed with 631 participants enrolled. Results have been announced in a press release by the company but have not been published yet. According to Amneal's website, the study successfully met its primary end point. Compared to immediate release LD/CD, IPX203 demonstrated statistically significant higher 'Good ON' time [0.53hr, $\mathrm{p}=0.0194)$ at 7 weeks. Post-hoc analysis showed increased 'good ON' time by $1.55 \mathrm{hr}(\mathrm{p}=<0.0001)$ per dose compared to active control by week 20 . The OFF time was reduced comparatively by $0.48 \mathrm{hr}(\mathrm{p}=0.0252)$. Amneal plans to file a new drug application in mid 2022 [4]. 
The Tavapadon studies, TEMPO-1,TEMPO-2, and TEMPO-3 are ongoing and recruiting participants. Exenatide is currently in recruiting phase as well.

Theravance Biopharma is evaluating ampreloxetine, a long-acting, once-daily norepinephrine reuptake inhibitor, as a potential treatment for symptomatic neurogenic orthostatic hypotension. There are three phase 3 trials designed around this molecule. SEQUOIA is a 4-week, multicentre, randomized, double-blind, placebocontrolled, parallel-group study of ampreloxetine (TD-9855) in treating symptomatic neurogenic orthostatic hypotension in subjects with dysautonomia in Multiple System Atrophy, PD, or Primary Autonomic Failure. REDWOOD is the next extension study followed by OAK, which is the open label safety and tolerability study [5]. SEQUOIA completed in June 2021 with 631 participants enrolled. Theravance biopharma announced negative results in a press release in September 2021 [6]. The study failed to meet its primary end point. Further data analysis is underway. The two sequential phase 3 trials continue to recruit but continuation is being reevaluated.

A summary of the status of trials reviewed as part of Phase 3 in Focus is shown in Table 8.

Table 8 - summary of status of trials reviewed as part of Phase 3 in Focus

\begin{tabular}{|c|c|c|c|c|c|}
\hline SPONSOR & AGENT & $\begin{array}{l}\text { REGISTRY } \\
\text { NUMBER }\end{array}$ & PHASE & $\begin{array}{l}\text { REGISTRY } \\
\text { STATUS }\end{array}$ & COMPLETION DATE \\
\hline NINDS & Isradipine & NCT02168842 & 3 & Completed & November 2018 \\
\hline Intec Pharma & Accordion Pill & NCT02605434 & 3 & Unknown & December 2019 \\
\hline Adamas Pharma & Gocovri EASE LID & NCT02136914 & 3 & Completed & December 2015 \\
\hline Adamas Pharma & Gocovri EASE LID 3 & NCT02274766 & 3 & Completed & 10 March 2016 \\
\hline Amneal & IPX203 & NCT03670953 & 3 & Completed & 15 June 2021 \\
\hline University College, London & Exenatide & NCT04232969 & 3 & Recruiting & 30 April 2024 (estimated) \\
\hline Cerevel Therapeutics & Tavapadon TEMPO 1 & NCT04201093 & 3 & Recruiting & October 2023 (estimated) \\
\hline Cerevel Therapeutics & Tavapadon TEMPO 2 & NCT04223193 & 3 & Recruiting & August 2023 (estimated) \\
\hline Cerevel Therapeutics & Tavapadon TEMPO 3 & NCT04542499 & 3 & Recruiting & March 2023 (estimated) \\
\hline Theravance Biopharma & Ampreloxetine SEQUOIA & NCT03750552 & 3 & Completed & 21 July 2021 \\
\hline Theravance Biopharma & $\begin{array}{l}\text { Ampreloxetine } \\
\text { REDWOOD }\end{array}$ & NCT03829657 & 3 & Recruiting & August 2022 (estimated) \\
\hline Theravance Biopharma & Ampreloxetine OAK & NCT04095793 & 3 & Recruiting & December 2025 \\
\hline
\end{tabular}

\section{References}

[1] Simuni T. Isradipine Versus Placebo in Early Parkinson Disease: A Randomized Trial. Annals of internal medicine. 2020;172(9):591-598. doi:10.7326/M19-2534

[2] Intec Pharma Reports Top-Line Phase 3 Trial Results of Accordion Pill-Carbidopa/Levodopa in Advanced Parkinson's Disease Patien - Bloomberg. Accessed November 24, 2021. https://www.bloomberg. $\mathrm{com} /$ press-releases/2019-07-22/intec-pharma-reports-top-line-phase-3-trial-results-of-accordion-pillcarbidopa-levodopa-in-advanced-parkinson-s-disease-patien

[3] McFarthing K, Prakash N, Simuni T. CLINICAL TRIAL HIGHLIGHTS - DYSKINESIA. Journal of Parkinson's Disease. 2019;9(3):449-465. doi:10.3233/JPD-199002 
[4] Amneal Pharmaceuticals, Inc. - Amneal Announces Positive Topline Results from Pivotal Phase 3 RISEPD Clinical Trial of IPX-203 in Patients with Parkinson's Disease Who Experience Motor Fluctuations. Accessed November 24, 2021. https://investors.amneal.com/news/press-releases/press-releasedetails/2021/Amneal-Announces-Positive-Topline-Results-from-Pivotal-Phase-3-RISE-PD-ClinicalTrial-of-IPX-203-in-Patients-with-Parkinsons-Disease-Who-Experience-Motor-Fluctuations/default. aspx

[5] McFarthing K, Prakash N, Simuni T. Clinical Trial Highlights: Phase 3 in Focus - Ampreloxetine, Theravance Biopharma. Journal of Parkinson's Disease. 2021;11(2):391-393. doi:10.3233/JPD-219004

[6] Theravance Biopharma, Inc. Announces Top-line Results from a Phase 3 Study of Ampreloxetine in Patients with Symptomatic Neurogenic Orthostatic Hypotension:: Theravance Biopharma. Accessed November 24, 2021. https://investor.theravance.com/news-releases/news-release-details/theravancebiopharma-inc-announces-top-line-results-phase-3 